\title{
Is there a relationship between pellet sugar content and schedule-induced polydipsia? A reexamination
}

\author{
JOHN A. FAIRBANK and ROBERT W. SCHAEFFER \\ Auburn University, Auburn, Alabama 36830
}

\begin{abstract}
Five groups of six food-deprived female albino rats received different formulas of Noyes sugared dry-food pellets under a FT 60 -sec food reinforcement schedule. Relative to baseline conditions that preceded and followed the FT schedule, 26 of 30 animals more than doubled their water consumption in the FT food-reinforcement schedule condition. The results of this study confirm previous investigators' reports of an inverse ordinal relationship between the amount of water consumed and sugar content of the food reinforcer, but this relationship was found to be neither numerically large nor statistically significant. It is concluded that premature theoretical speculation, drawn from numerical relationships that lack statistical significance, may be a very unprofitable technique for identification of underlying causes of scheduleinduced polydipsia.
\end{abstract}

Falk (1961) was the first investigator to report that, under certain intermittent schedules of dry-food reinforcement, food-deprived rats drank copious amounts of concurrently available water. In the nearly two decades since Falk's (1961) initial report, more than 200 investigations of this remarkable drinking phenomenon, commonly referred to as schedule-induced polydipsia (SIP), have been undertaken (Christian, Schaeffer, \& King, 1977). Several investigators (Christian, Riester, \& Schaeffer, 1973; Christian \& Schaeffer, 1973; Colotla $\&$ Keehn, 1975) have suggested that the concentration of sugar in dry-food reinforcers significantly affects the acquisition and maintenance of SIP in rats. In the first study to examine this relation (Christian \& Schaeffer, 1973), five groups of food-deprived rats that received different formulas of $45-\mathrm{mg}$ Noyes pellets containing $0 \%$ sugar, $7.5 \%$ glucose, or $4.0 \%, 16.0 \%$, or $32.0 \%$ sucrose, by weight, were tested under a FT 60 -sec schedule of dry-food reinforcement. It was reported that the subjects in these five groups developed SIP and that a modest, inverse, ordinal relationship between water intake levels and sucrose concentrations of dry. food pellets was evident. In addition, it was noted that the group that received sugarless pellets drank more water than the group that received pellets containing 7.5\% glucose, and that both of the preceding groups drank more than any of the groups that received sucrose pellets.

Unfortunately, several partial replications of Christian and Schaeffer's (1973) experimental design have failed to completely corroborate their findings. For example,

The data upon which this report is based were submitted by the senior author under the direction of the junior author in partial fulfillment of the thesis requirement for the master of science degree at Auburn University.
Christian et al. (1973), in a partial replication of Christian and Schaeffer's (1973) procedure, found that sugared pellets produced less drinking than sugarless pellets, but failed to confirm the ordered inverse relationship between pellet sucrose concentration and SIP reported by Christian and Schaeffer (1973). In a study that employed a Graeco-Latin square experimental design, and in which subjects were presented with counterbalanced combinations of four different fluids and sugarless, $7.5 \%$ glucose, and $4 \%$ and $32 \%$ sucrose pellets, Colotla and Keehn (1975) obtained the same inverse relationship between sucrose concentration of dry-food pellets and SIP that had been reported by Christian and Schaeffer (1973). In contradistinction to the findings of Christian and his colleagues (Christian et al., 1973; Christian \& Schaeffer, 1973), however, Colotla and Keehn (1975) reported greater water intakes for sugared pellets than for sugarless pellets.

Inasmuch as the foregoing partial replications (Christian et al., 1973; Colotla \& Keehn, 1975) of Christian and Schaeffer's (1973) procedures have produced mixed findings, a reexamination of the significance of the relationship between water intake and pellet sugar composition is of interest. The present study, a direct replication of the experimental design employed by Christian and Schaeffer (1973), reexamined the relationship between SIP and pellet sugar concentration, using multiple parametric tests rather than nonparametric tests, and larger groups of subjects than have been used in any of the previous studies described above.

\section{METHOD}

\section{Subjects}

The subjects were 30 experimentally naive, female albino rats, about 90 days old at the start of the experiment. Subjects 
were individually housed in Wahmann LC 175-C75 cages under conditions of constant illumination and relatively constant temperature and humidity. All experimental subjects were reduced to $85 \%$ of their free-feeding weight and maintained at that level throughout the experiment. At all times water was freely available to subjects in the home cage and the experimental chamber.

\section{Apparatus}

Ten Lehigh Valley Electronics (LVE) standard experimental small animal chambers were employed throughout the study. The test chambers were modified by removing the right lever to permit the insertion of a standard Wahmann stainless steel drinking tube. Recording of licks and deliveries of Noyes $45-\mathrm{mg}$ dry-food pellets and all programming were automatically accomplished by a Data General Nova computer and standard electromechanical circuitry. A graphic record of the temporal pattern of lick responses and pellet deliveries for each session was made on Gerbrands cumulative recorders. Water intakes during each experimental session were determined by weighing the water bottles before and after each experimental session and converting these values to milliliters.

\section{Procedure}

The 30 subjects were randomly assigned to five groups, with 6 subjects in each group. Each group received a different $45-\mathrm{mg}$ Noyes dry-food pellet during the experiment: Group 1 received Noyes $45-\mathrm{mg}$ standard formula pellets; Group 2 received Noyes 45-mg sugarless pellets without the $7.5 \%$ glucose binder; Group 3 received Noyes $45-\mathrm{mg}$ sugarless pellets with $4 \%$ sucrose added, by weight; Group 4 received Noyes $45-\mathrm{mg}$ sugarless pellets with $16 \%$ sucrose added; and Group 5 received Noyes 45 -mg sugarless pellets with $32 \%$ sucrose added.

The between-groups experimental design consisted of three phases: baseline (A), experimental (B), and reinstatement of baseline (A). During the initial baseline phase, 100 of the appropriate formula dry-food pellets were placed in the food magazine. The subjects remained in the test chambers for $100 \mathrm{~min}$, during which licks and water intakes were recorded. The empty pellet dispenser was also operated on a FT 60-sec schedule during the baseline phase. The initial baseline phase continued until water intakes in the test chamber stabilized and the subjects regularly consumed all food pellets.

In the experimental phase, all subjects received 100 of the appropriate formula Noyes pellets on a FT $60-\mathrm{sec}$ schedule of pellet delivery. Daily session time was $100 \mathrm{~min}$, the length of time required to dispense all 100 pellets. The experimental phase remained in effect until water intakes stabilized for each animal. In the third phase of the experiment, the conditions of the initial baseline phase were reinstated.

Water intake stability criteria for all phases required that the differences between the total intakes of the last three and previous three test sessions be less than $20 \%$ of the total intake of the last six sessions. The criterion had to be met by all 30 animals before termination of any experimental phase or introduction of a new experimental phase.

\section{RESULTS}

Group mean water intakes for the five pellet formula groups, across all experimental conditions, are presented in Figure 1. Inspection of this figure reveals that relative to pre- and postexperimental baseline conditions, all groups greatly increased their fluid intakes in the experimental FT food-reinforcement schedule condition. A twofold or greater increase in water intake during the experimental condition, relative to water intake in the

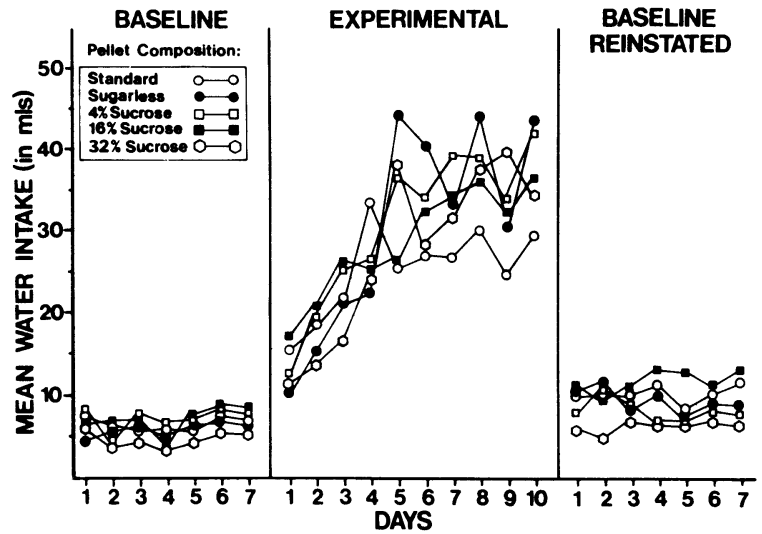

Figure 1. Group mean milliliters drunk as a function of pellet sugar content in all three phases of the experiment.

preexperimental baseline condition, (Christian et al., 1977), was employed as the criterion for defining SIP. Of the 30 animals in the five groups, only four subjects failed to more than double their water intake in the experimental phase. Specifically, Group 1 contained two subjects and Groups 4 and 5 each contained one subject that failed to meet the criterion defining SIP. During the postexperimental return to baseline phase, the mean water intake levels obtained in the initial baseline phase were recovered for all groups and all subjects.

Examination of the mean group data points that were obtained during the experimental phase suggests a modest, inverse ordinal relationship between sugar content of the dry-food reinforcer and the amount of water consumed. It must be noted that there was marked heterogeneity of between-group values and some instability of group mean values across sessions within the experimental phase. Nonetheless, the mean values obtained appear to add confirmation to previous investigators' (Christian \& Schaeffer, 1973; Colotla \& Keehn, 1975) reports of an inverse ordinal relationship between SIP and the sugar content of the dry food pellet.

In order to determine whether these numerically suggestive between-phases and between-groups differences in mean water intake were statistically significant, the following statistical analyses were performed. First, a three-way analysis of variance, using all subjects in all groups, with groups as the first factor, phases as the second factor, and trials as the third factor, was applied to the data from the last 6 days of each phase. There was a significant difference between phases $[F(2)=69.65$, $p<.01$ ], confirming the statistical significance of the obvious numerical differences in water intake between the baseline and experimental phases shown in Figure 1. However, the difference between groups in this statistical analysis was not significant $[F(4)=.34, p>.05]$. Next, a two-way analysis of variance, using all subjects in all groups, with groups as the first factor and trials as the second factor, was performed, using only the data from the last six sessions of the experimental phase. 
This analysis also failed to identify statistically significant differences between groups $[F(4)=.43, p>.05]$. Since these first two statistical analyses included all of the data obtained in the experiment, including that from the four nonpolydipsic subjects, it was decided that a third analysis should be performed, but only on the polydipsic animals. This analysis was conducted to permit examination of possible group differences that might have been obscured by any possible contamination effects of the data that had been obtained from the nonpolydipsic animals. The third computation, a twoway analysis of variance with groups as the first factor and trials as the second factor, was performed only on data corrected to exclude the data from the four nonpolydipsic subjects. Since Group 1 contained two subjects that failed to meet the criterion defining SIP, it was decided to perform the analysis using only four polydipsic subjects per group. Since Groups 4 and 5 each contained but one nonpolydipsic subject, a random selection procedure was used to remove one additional subject from each of these two groups. Since all subjects in Groups 2 and 3 developed SIP, two subjects from each of these groups were identified by a random selection procedure and discarded. Thus, the third statistical analysis was performed on the data obtained from 20 polydipsic subjects, with four subjects retained in each of the five formula groups. Once again, the difference between groups was not significant $[F(4)=.04, p>$ .05 ].

As will be evident to the reader, the statistical analyses described above permitted, successively, the examination of the effects of pellet formulas upon water intakes both with and without the effects of nonpolydipsic subjects entering into the analyses. Because none of the three analyses showed pellet composition to significantly affect water intake, the ordinal relationship between sugar content of dry-food reinforcer and water intake that might be inferred from the mean data in Figure 1 is, if actually a genuine phenomenon, at best a very weak relationship.

\section{DISCUSSION}

In the present experiment, it was found that mean water intakes were ordered in the same general numerical relation reported by previous investigators (Christian \& Schaeffer, 1973; Colotla \& Keehn, 1975). However, the ordinal inverse relationship between sugar content of the dry-food reinforcer and water intake was neither numerically large nor statistically significant. Previous investigators (Christian, 1976; Christian \& Schaeffer, 1973; Christian et al., 1973) have suggested that the modest relations they have found between water intake and pellet composition may be attributable to frustration-induced motivational correlates of pellet sugar composition. However, the present study, which used larger groups of subjects and more exhaustive parametric statistical analyses than have previously been reported, demonstrated that this relationship lacks statistical significance and, consequently, probably also lacks true numerical importance. Relative to the statistically significant between-groups effects in water ingestion that are consistently produced by experimental parameters such as the type of reinforcement schedule employed (Falk, 1971) and type of concurrently available fluid proffered (Ten Eyck \& Schaeffer, 1969), the relationship between pellet composition and water intake appears to be relatively inconsequential. Given the failure to find a statistically significant relation between these two parameters in the present study, and the inconsistent betweenexperimenters effects found by others who have looked at pellets and water consumption, the relationship between pellet composition and amount drunk might most appropriately be viewed at this time as no relationship, rather than a potentially revealing correlate of significant motivational states. As such, premature theoretical speculation, drawn from numerical relationships that lack statistical significance, may not only be a very unprofitable technique for identification of underlying causes of SIP, but may be misleading.

\section{REFERENCES}

Christian, W. P. Control of schedule-induced polydipsia: Sugar content of the dry food reinforcer. Psychological Record, 1976, 26, 41-47.

Christian, W. P., Riester, R. W., \& Schaeffer, R. W. Effects of sucrose concentrations upon schedule-induced polydipsia using free and response-contingent dry-food reinforcement schedules. Bulletin of the Psychonomic Society, 1973, 2, 65-68.

Christian, W. P., \& Schaeffer, R. W. Effects of sucrose concentrations upon schedule-induced polydipsia on a FFI60-sec dry-food reinforcement schedule. Psychological Reports, 1973, 32, 1067-1073.

Christian, W. P., Schaeffer, R. W., \& King, G. D. Scheduleinduced behavior: Research and theory. Montreal: Eden, 1977.

Colotla, V. A., \& KeEHN, J. D. Effects of reinforcer-pellet composition on schedule-induced polydipsia with alcohol, water, and saccharin. Psychological Record, 1975, 25, 91-98.

FALK, J. L. Production of polydipsia in normal rats by an intermittent food schedule. Science, 1961, 133, 195-196.

FALK, J. L. Theoretical review: The nature and determinants of adjunctive behavior. Physiology \& Behavior, 1971, 6, 577-588.

Ten Eyck, R. L., \& ScuAEFfER, R. W. Instrumental and motivationally induced drinking under dry food reinforcement schedules. Psychological Reports, 1969, 24, 991-998.

(Received for publication May 1, 1978.) 